\title{
Rational QR-iteration without inversion
}

\section{Raf Vandebril, Marc Van Barel and Nicola Mastronardi}

\author{
Raf Vandebril \\ Department of Computer Science \\ KU Leuven, Belgium \\ Raf.Vandebril@cs.kuleuven.be \\ Marc Van Barel \\ Department of Computer Science \\ KU Leuven, Belgium \\ Marc.VanBarel@cs.kuleuven.be \\ Nicola Mastronardi \\ Istituto per le Applicazioni del Calcolo \\ M. Picone, sez. Bari, Italy \\ N.Mastronardi@ba.iac.cnr.it
}

\begin{abstract}
In this manuscript a new method will be presented for performing a $Q R$-iteration with $(A-\sigma I)(A-\kappa I)^{-1}=Q R$ without explicit inversion of the factor $(A-\kappa I)^{-1}$. A $Q R$-method driven by a rational function is attractive since convergence can occur at both sides of the matrix.

Each step of this new iteration consists of two substeps. In the explicit version, first an $R Q$-factorization of the initial matrix $A-\kappa I=R Q$ will be computed, followed by a $Q R$-factorization of the matrix $(A-\sigma I) Q^{H}$. The factorization of $(A-\sigma I) Q^{H}$ can be computed in an intelligent manner, exploiting properties of the already known $R Q$-factorization of $A-\kappa I$. The similarity transformation yielding the $Q R$-step is defined by the unitary factor $Q$ in the $Q R$-factorization of the transformed matrix $(A-$ $\sigma I) Q^{H}$.

Examples will be given, illustrating how to efficiently compute the factorization for some specific classes of matrices. The novelties of this approach with respect to these matrix classes will be discussed.
\end{abstract}

\section{Article information}

- Vandebril, Raf; Van Barel, Marc; Mastronardi, Nicola. Rational QR-iteration without inversion, Numerische Mathematik, volume 110, issue 4, pages 561-575, 2008.

- The content of this article is identical to the content of the published paper, but without the final typesetting by the publisher.

- Journal's homepage: http://link.springer.com/journal/211

- Published version: http://dx.doi.org/10.1007/s00211-008-0177-3

- KU Leuven's repository url: https://lirias.kuleuven.be/handle/123456789/256357

\section{KU LEUVEN}




\title{
Rational $Q R$-iteration without inversion *
}

\author{
Raf Vandebril $^{1}$, Marc Van Barel ${ }^{1}$, Nicola Mastronardi ${ }^{2}$ \\ 1 KULeuven, Dept. of Computer Science, 3001 Leuven(Heverlee) \\ e-mail: $\{$ raf.vandebril, marc.vanbarel\}ecs.kuleuven.be \\ 2 Istituto per le Applicazioni del Calcolo M. Picone, sez. Bari, \\ e-mail: mastronardieba.iac.cnr.it
}

October 13, 2011

\begin{abstract}
In this manuscript a new method will be presented for performing a $Q R$-iteration with $(A-\sigma I)(A-\kappa I)^{-1}=Q R$ without explicit inversion of the factor $(A-\kappa I)^{-1}$. A $Q R$-method driven by a rational function is attractive since convergence can occur at both sides of the matrix.

Each step of this new iteration consists of two substeps. In the explicit version, first an $R Q$ factorization of the initial matrix $A-\kappa I=R Q$ will be computed, followed by a $Q R$-factorization of the matrix $(A-\sigma I) Q^{H}$. The factorization of $(A-\sigma I) Q^{H}$ can be computed in an intelligent manner, exploiting properties of the already known $R Q$-factorization of $A-\kappa I$. The similarity transformation yielding the $Q R$-step is defined by the unitary factor $Q$ in the $Q R$-factorization of the transformed matrix $(A-\sigma I) Q^{H}$.

Examples will be given, illustrating how to efficiently compute the factorization for some specific classes of matrices. The novelties of this approach with respect to these matrix classes will be discussed.
\end{abstract}

Key words $Q R$-algorithm, eigenvalues, rational function

\section{Introduction and preliminary results}

If one wants to compute all eigenvalues (and corresponding eigenvectors) of a matrix $A$, quite often the standard $Q R$-method is used. For a certain matrix $A$, and a specific shift $\sigma$ we obtain the following equations, determining the $Q R$-method:

$$
\begin{aligned}
A-\sigma I & =Q R, \\
\hat{A} & =R Q+\sigma I=Q^{H} A Q,
\end{aligned}
$$

where $\hat{A}$ yields the new iterate. When applying several steps of the $Q R$-method with well-chosen shifts $\sigma$, the method will converge to an upper triangular matrix containing the eigenvalues of the original matrix $A$ (see, e.g., [1,2]). When applying the (multishifted) $Q R$-method (or a general $G R$ method) convergence typically occurs at the bottom of the matrix. A variant of the $Q R$-method is the $R Q$-method yielding similar properties, but convergence will now occur at the top of the matrix. The challenge is to combine both methods to obtain convergence at both ends of the

\footnotetext{
* The research of the first two authors, was partially supported by the Research Council K.U.Leuven, project OT/05/40 (Large rank structured matrix computations), CoE EF/05/006 Optimization in Engineering (OPTEC), by the Fund for Scientific Research-Flanders (Belgium), G.0455.0 (RHPH: Riemann-Hilbert problems, random matrices and Padé-Hermite approximation), G.0423.05 (RAM: Rational modelling: optimal conditioning and stable algorithms), and by the Interuniversity Poles of Attraction Poles, initiated by the Belgian State, Science Policy Office, Belgian Network DYSCO (Dynamical Systems, Control, and Optimization). The first author has a grant as "Postdoctoraal Onderzoeker" from the Fund for Scientific Research-Flanders (Belgium). The work of the third author was partially supported by MIUR, grant number 2004015437. The scientific responsibility rests with the authors.
} 
matrix. This results in choosing a suitable function $f($.$) in the matrix A$, such that the unitary transformation $Q$ from $f(A)=Q R$ will yield these properties. It will be shown that $f(A)=(A-$ $\sigma I)(A-\kappa I)^{-1}$ fulfills this role. Unfortunately the inversion of the factor $(A-\kappa I)$ is numerically a bad idea, especially since one will choose the shift $\kappa$ as close as possible to an eigenvalue of the matrix $A$, resulting therefore in an almost singular $(A-\kappa I)$.

In this paper we will present a numerically stable manner for computing the factorization $(A-\sigma I)(A-\kappa I)^{-1}$, without inversion. In this introduction, some basic facts about $Q R$-algorithms, both implicit and explicit versions as well as their convergence analysis will be discussed. We assume, however, that the reader is quite familiar with these methods, their convergence analysis and so forth. Well-known results will not be discussed in great detail, references will be presented.

In Equation (1), the matrix $\hat{A}$ can be computed in two different ways, the first manner is referred to as the explicit $Q R$-method, whereas the formula $Q^{H} A Q$ can lead to an implicit approach (see, e.g., $[3,4]$ ). The new algorithm presented in this manuscript admits both types of methods. We will not design an implicit method, as it involves a lot of technical details, and heavily depends on the matrix class considered. In practice, however, implicit methods are often preferrable above the explicit versions. In this manuscript we only derive the new algorithm and we do not restrict ourselves to any specific class of matrices. Few comments will however be presented on how to implement an implicit version.

Important for all $Q R$-related methods is the preservation of the structure, i.e., one wants the matrices resulting from a step of the $Q R$-method to have the same structure as the original matrix, such as, e.g., Hessenberg. We will show that any matrix structure invariant under the $Q R$-method will also be preserved under the $Q R$-iteration driven by a rational function.

The convergence of the $Q R$-algorithm, and all its variants (generically called $G R$-algorithms ${ }^{1}$ $[5,6])$ can be interpreted as subspace iteration, determined by polynomials in the matrices $A$, followed by a coordinate transformation. Standard polynomial forms for one step of the standard $Q R$-algorithm look like $p(\lambda)=\lambda-\sigma$, corresponding to the single shift strategy and $p(\lambda)=$ $\left(\lambda-\sigma_{1}\right)\left(\lambda-\sigma_{2}\right)$ corresponding to the double shift strategy. Based on these polynomials one can derive bounds on the convergence of $G R$-algorithms. A similar result will be provided for the $Q R$-iteration driven by a rational function.

The cost of any kind of $Q R$-iteration is mostly determined by the structure of the unitary matrix $Q$, coming from the $Q R$-factorization of the matrix $A-\sigma I$. Hence, we will derive for some examples the structure of the resulting unitary factor $Q$. The complexity of this factor will be compared to the complexity of the unitary factor coming from a single shifted $Q R$-step.

The manuscript is organized as follows: in the first section the new iteration for performing a $Q R$-step driven by a rational function will be described. In Section 3 we will comment on some $Q R$-related issues, such as the preservation of the structure and how to perform the implicit $Q R$ steps. In Section 4 some examples will be given. Finally, conclusions and future work will be presented.

\subsection{Rational Krylov methods}

Most of the iterative methods for computing eigenvalues of structured/sparse matrices are based on the so-called Krylov subspaces. Given a matrix $A$ whose eigenvalues one would like to compute and an initial vector $\mathbf{v}$ we have the following Krylov subspace

$$
\mathcal{K}^{j}(A, \mathbf{v})=\left\{\mathbf{v}, A \mathbf{v}, \ldots A^{j-1} \mathbf{v}\right\} .
$$

The idea of using rational functions in $A$ instead of the standard powers of $A$ can be found in [7]. The idea is to work with the following Krylov sequence, in which all $\varphi_{i}(\lambda)$ are rational functions in $\lambda$ :

$$
\mathcal{F}^{j}(A, \mathbf{v})=\left\{\varphi_{1}(A) \mathbf{v}, \varphi_{2}(A) \mathbf{v}, \ldots \varphi_{j}(A) \mathbf{v}\right\} .
$$

1 This covers a more general class of iterations such as $Q R$-methods, $L R$-methods and so forth. 
The idea in Rational Krylov methods is to choose the functions in an intelligent way, to speed up the convergence of the iterative methods.

Similarly one chooses a good shift to speed up convergence in case of the $Q R$-method. In this manuscript we will develop a technique to perform $Q R$-steps driven by a rational function onto matrices, without explicit inversion.

Information on rational Krylov methods can be found in, e.g., [7,8,9]. Moreover, when considering a tridiagonal matrix $T$ and the standard Krylov subspace, there is well-known relation between the $Q R$-factorization of the matrix $T$ and this Krylov sequence (see [4]). Similarly there is a well-known relation between rational Krylov sequences and semiseparable plus diagonal matrices (see [10]).

\section{A $Q R$-iteration driven by a rational function}

$Q R$-methods based on rational functions are already in use for computing eigenvalues of e.g. Hamiltonian (symplectic) matrices $[11,12,13]$. The basic idea is to perform an $R Q$-iteration onto $(A-\kappa I)$ and a $Q R$-iteration onto $Q A Q^{H}$. Implicit versions of this method exist and create bulges at both ends of the matrix resulting in bidirectional chasing algorithms $[14,12]$. The approach we propose is significantly different.

\subsection{The iteration}

Let us consider the matrix $A$, whose eigenvalues we would like to compute, by using the rational iteration. Assume the shifts $\kappa$ and $\sigma$ to be known. Since we want the shifts to approximate eigenvalues of the original matrix, inverting the matrix $A-\kappa I$ is not an option. Hence, the following technique is used.

Given

$$
A-\kappa I=R Q,
$$

we obtain

$$
\begin{aligned}
A-\sigma I & =\left((A-\kappa) Q^{H}-(\sigma-\kappa) Q^{H}\right) Q \\
& =\left(R-(\sigma-\kappa) Q^{H}\right) Q .
\end{aligned}
$$

Computing now the $Q R$-factorization of the matrix $R-(\sigma-\kappa) Q^{H}$, and substituting this result in the equations above leads to the following result:

$$
\begin{aligned}
\hat{Q} \hat{R} & =R-(\sigma-\kappa) Q^{H}, \\
A-\sigma I & =\hat{Q} \hat{R} Q .
\end{aligned}
$$

Instead of having computed a $Q R$-factorization of the matrix $A-\sigma I$, we have computed an $U R V$ decomposition, in which the unitary factor $V$ was determined beforehand.

Applying now the following unitary similarity transformation onto the matrix $A$ gives us the next iterate:

$$
\hat{A}=\hat{Q}^{H} A \hat{Q} .
$$

Based on matrix $\hat{A}$ we can continue with our iteration process.

A straightforward computation reveals that

$$
(A-\sigma I)(A-\kappa I)^{-1}=\hat{Q} \hat{R} R^{-1} .
$$

Hence the unitary matrix $\hat{Q}$ determines a $Q R$-step onto the matrix $A$ driven by a rational function.

It might seem at first that this iteration is twice as expensive as a single shifted $Q R$-step since both a $Q R$ and an $R Q$-factorization need to be computed. We will show later on that this is not completely true since an implicit method is only determined by one unitary transformation, which is the matrix $\hat{Q}$ in this case. This matrix $\hat{Q}$ can be more complex than in the single shifted $Q R$-case, but in several examples the matrix will not necessarily be more complex, in few cases it is even of a more simple form. Moreover, in implicit versions, often only the first column of $\hat{Q}$ is necessary. 


\subsection{Non-unitary factorizations}

We have always assumed to be working with unitary transformations. Without any loss of generality one can use non-unitary transformations. Suppose we have the following equations (with $G$ and $\hat{G}$ invertible, not necessarily unitary, matrices):

$$
A-\kappa I=R G,
$$

for which $R$ is an upper triangular matrix. This will be used in the following equations:

$$
\begin{aligned}
A-\sigma I & =\left((A-\kappa) G^{-1}-(\sigma-\kappa) G^{-1}\right) G \\
& =\left(R-(\sigma-\kappa) G^{-1}\right) G, \\
A-\sigma I & =\hat{G} \hat{R} G .
\end{aligned}
$$

The matrix $\hat{R}$ is again upper triangular. The following similarity transformation is then determined by the matrix $\hat{G}$ :

$$
\hat{A}=\hat{G}^{-1} A \hat{G} .
$$

This brings us back to the case of $G R$-algorithms (see [5]). Unfortunately loosening the unitarity constraint can result in the use of ill conditioned matrices. One can also use a combination of unitary and non-unitary transformations. For example using a non-unitary transformation $G^{(1)}$ and a unitary $\hat{G}^{(1)}$ still determines a unitary similarity transformation onto the matrix $A^{(1)}$, instead of simply a similarity transformation. This creates flexibility in the design of the method.

Even though most of the results mentioned in the remainder are valid for general $G R$-methods, we will restrict ourselves to the unitary case.

\section{$3 Q R$-related issues}

In this section we will discuss the preservation of structure and how to design an implicit $Q R$ method.

\subsection{Preservation of the structure}

Essential in effective $Q R$-algorithms, for maintaining a low cost iteration, is the preservation of the structure under a step of the $Q R$-method.

Suppose a matrix $A$ is given, whose structure is preserved under an iteration of the $Q R$ algorithm. Any kind of structure can be considered such as Hessenberg [15,16], tridiagonal [2], band, Hessenberg-like [17], semiseparable, quasiseparable [18], unitary plus low rank [19,20,21], etc. $^{2}$

Similar to the $Q R$-case one can prove that a step of the $R Q$-algorithm preserves the structure of the matrix $A$.

It is known that the unitary matrix $\hat{Q}$ determining the similarity transformation satisfies the following relation

$$
(A-\sigma I)(A-\kappa I)^{-1}=\hat{Q} \hat{R} R^{-1} .
$$

We want to prove that $\hat{A}=\hat{Q}^{H} A \hat{Q}$ has the same structure as the original matrix $A$.

It is known that the equation above can be decomposed into two steps. In a first step one computes the $R Q$-factorization of the matrix $(A-\kappa I)$, and performs a step of the $R Q$-method

$$
\begin{aligned}
(A-\kappa I) & =R Q, \\
\tilde{A} & =Q A Q^{H} .
\end{aligned}
$$

\footnotetext{
2 Even though not mentioned, in case of a band matrix, a tridiagonal matrix, ... we implicitly assume the matrices to be symmetric.
} 
The matrix $\tilde{A}$ inherits the structure of the matrix $A$. In the second step a $Q R$-step is performed on the matrix $\tilde{A}$. We obtain:

$$
\begin{aligned}
(\tilde{A}-\sigma I) & =\tilde{Q} \tilde{R}, \\
\tilde{\hat{A}} & =\tilde{Q}^{H} \tilde{A} \tilde{Q}=\tilde{Q}^{H} Q A Q^{H} \tilde{Q}
\end{aligned}
$$

The single shifted $Q R$-method states now that the matrix $\hat{\tilde{A}}$ satisfies the same structural constraints as $\tilde{A}$ and hence of $A$. When $\hat{\tilde{A}}$ is essentially equivalent to $\hat{A}$, the statement is proved.

The connection between the $Q R$-factorization of $(A-\sigma I)(A-\kappa I)^{-1}$ and the above results is the following:

$$
\begin{aligned}
\hat{Q} \hat{R} R^{-1}=(A-\sigma I)(A-\kappa I)^{-1} & =(A-\sigma I) Q^{H} R^{-1} \\
& =Q^{H} Q(A-\sigma I) Q^{H} R^{-1} \\
& =Q^{H}(\tilde{A}-\sigma I) R^{-1} \\
& =Q^{H} \tilde{Q} \tilde{R} R^{-1} .
\end{aligned}
$$

It is clear that the matrices $\hat{Q}$ and $Q^{H} \tilde{Q}$ are essentially the same, hence they determine essentially the same similarity transformation. As a result the structure of the matrix $A$ is preserved under the similarity transformation determined by the matrix $\hat{Q}$, from Equation (2).

\subsection{Implicit and explicit version}

It might seem at first that the presented method converges twice as fast since convergence occurs at both ends of the matrix, but is also twice as expensive. Twice as expensive, because one has to compute two factorizations: one $Q R$ - and one $R Q$-factorization to fully determine the complete $Q R$-step.

In an explicit version of the method it is true that one has to compute two full factorizations. One has to compute the complete matrix $Q$ to determine the next iterate. Implicit methods, in general, do not need the full matrix $Q$. Only the first column $Q \mathbf{e}_{1}$ is sufficient for determining the iteration in a unique way.

An implicit $Q R$-method performed onto the structured matrix $A$, has only few building blocks (see also $[3,4,22]$ ):

- Determine the first column of the following matrix product $\left(A-\sigma_{i}\right)\left(A-\kappa_{i}\right)^{-1}$, i.e., determine $\mathbf{v}=\left(A-\sigma_{i}\right)\left(A-\kappa_{i}\right)^{-1} \mathbf{e}_{1}$.

- Determine now a unitary transformation $W_{1}$, either a single Householder transformation, or few Givens transformations, such that $W_{1}^{H} \mathbf{v}=\|\mathbf{v}\| \mathbf{e}_{1}$.

- Apply this transformation to the matrix $A$ :

$$
\tilde{A}=W_{1}^{H} A W_{1} .
$$

The result of this transformation is a new matrix $\tilde{A}$, which does in general not meet the structural constraints posed on the matrix $A$, anymore.

- Restore the structure of the matrix $\tilde{A}$, by a unitary similarity transformation $W_{2}$, such that $W_{2} \mathbf{e}_{1}=\mathbf{e}_{1}$. As a result we obtain the matrix:

$$
\hat{A}=W_{2}^{H} \tilde{A} W_{2} .
$$

- Using a kind of implicit $Q$-theorem, one can prove that the result of this high-level algorithm gives us the matrix $\hat{A}$, which is essentially the same as the matrix resulting from a step of the $Q R$-method onto the matrix $A$.

One can conclude that in a certain sense only the first column of the matrix $Q$ determines the unitary similarity transformation, yielding a $Q R$-step.

Applying the technique mentioned above can lead to a fast implementation for the $Q R$-method driven by a rational function, since only the first column of the matrix $Q$ is needed. It is shown 
in [23] that the vector $\mathbf{v}$ determining the $Q R$-step for semiseparable matrices is even more simple than the vector $\mathbf{v}$ from the $Q R$-method applied onto the semiseparable matrix.

Since an implicit technique also involves determining the similarity transformation $W_{2}$, and this is dependent on the structure of the matrix, we will not design implicit techniques here. In the forthcoming section we will, however, show some examples of matrices for which the algorithm might result in a low complexity algorithm, with an advantageous convergence behavior.

\subsection{Convergence}

Since the unitary matrix determining the similarity transformation is coming from the $Q R$-factorization of a rational function in $A$, standard convergence theory [5] applies based the rational function $p(\lambda)=(\lambda-\sigma)(\lambda-\kappa)^{-1}$.

The manuscript [5] provides general subspace iteration results applicable, with minor changes, to this case. The shift $\kappa$ close to an eigenvalue creates convergence at the top. The shift $\sigma$ close to an eigenvalue creates convergence at the bottom. Standard analysis predicting the convergence speed applies independently to both ends of the matrix, in case the shifts are well separated. Hence a good choice of shifts would be $\kappa$ close to the largest eigenvalue and $\sigma$ close to the smallest eigenvalue.

\section{Examples}

In this section we will apply the new iteration onto some classes of matrices and comment on the structure of the intermediate matrices and especially on the unitary matrix $Q$.

A combination of the single shifted $R Q$-method followed by a single shifted $Q R$-method results in fact in performing twice an iteration. Hence, one might expect the unitary factor $Q$ determining the new similarity transformation to be twice as complex as the unitary factor coming from one step of the $Q R$ or $R Q$-method. Since the unitary matrix mostly determines the complexity of the resulting $Q R$-method, its structure is quite important.

We will show, that based on the specific structure of the following matrices the new iteration does not necessarily results in more complex unitary matrices. This means that the new approach might result for several cases into more efficient algorithms, since the corresponding unitary matrix is of simple form. For simplicity we start with the semiseparable case having shift $\kappa=0$. This is followed by the generic semiseparable (plus diagonal) case having shift $\kappa \neq 0$.

\subsection{Semiseparable matrices}

Semiseparable matrices are suitable for the new $Q R$-iterate. Let us comment on the case $\kappa_{i}=0$. With suitable we mean that the resulting unitary factor $Q$, will be more easy representable than the unitary factor from the single shifted $Q R$-iteration. Remark that, we will not take the extra convergence behavior into consideration. Only the mathematical properties of performing a single step of the new method are discussed.

Let us define the class of semiseparable matrices.

Definition 1 A square matrix $S$ is called a semiseparable matrix if the following relations are satisfied:

$$
\operatorname{rank}(S(1: i, i: n)) \leq 1 \text { and } \operatorname{rank}(S(i: n, 1: i)) \leq 1 \text {, }
$$

for all feasible $i$.

More precisely this means that a matrix has all subblocks taken out of the matrix below the superdiagonal of rank 1 (a similar remark holds for the upper triangular part). For example the 
elements $\nabla$ in the following matrix, make up the lower triangular semiseparable part of a semiseparable matrix. This means that all submatrices taken out of the part of the matrix marked with $\square$ are of rank at most one:

$$
S=\left(s_{i j}\right)_{i j}=\left[\begin{array}{l}
\nabla \times \times \times \times \\
\nabla \nabla \times \times \times \\
\nabla \nabla \nabla \times \times \\
\nabla \nabla \nabla \nabla \times \\
\nabla \nabla \nabla \nabla \nabla
\end{array}\right] .
$$

Each nonsingular semiseparable matrix its inverse is tridiagonal and vice versa.

Let us briefly recall the $Q R$-factorization of semiseparable plus diagonal matrices.

4.1.1 The QR-factorization of a semiseparable plus diagonal matrix Suppose $S$ to be of semiseparable form. To perform a $Q R$-iteration, the $Q R$-factorization of $S-\sigma I$ is desired. Without loss of generality we assume to be working with a general semiseparable plus diagonal matrix now: $S+D$, with $D$ diagonal.

The $Q R$-factorization will consist of $2 n-2$ Givens transformation, for an $n \times n$ matrix $S$ [24, $25,26]$.

Since the last two rows of the matrix $S$ are linearly dependent up to the diagonal, a single Givens transformation acting on rows $n-1$ and $n$ can annihilate all elements up to the diagonal in the last row. This transformation is followed by a Givens transformation acting on row $n-2$ and $n-1$, annihilating all elements up to the diagonal in row $n-1$. Continuing this procedure (see, e.g., [24]) results in an upper triangular matrix:

$$
G_{n-1}^{H} G_{n-2}^{H} \ldots G_{1}^{H} S=\tilde{R} .
$$

Since the $Q R$-factorization of $S+D$ is desired some extra operations are needed to compute the $Q R$-factorization of the following resulting Hessenberg matrix $H$ :

$$
G_{n-1}^{H} G_{n-2}^{H} \ldots G_{1}^{H}(S+D)=H .
$$

Computing the $Q R$-factorization of the Hessenberg matrix involves again a sequence of $n-1$ Givens transformations. The first Givens transformation acts on row 1 and 2 and annihilates the subdiagonal element $H_{21}$. The second Givens transformation acts on row 2 and 3 and annihilates the second subdiagonal element. Finally we obtain:

$$
G_{2 n-2}^{H} G_{2 n-3}^{H} \ldots G_{1}^{H}(S+D)=R .
$$

Hence the unitary matrix $Q$ from the $Q R$-factorization of the semiseparable plus diagonal matrix $S+D$ consists of a product of $2 n-2$ Givens transformations. These $2 n-2$ transformations are effectively needed when performing a step of the $Q R$-method onto semiseparable matrices (see, e.g., [17]).

4.1.2 Computing the new iteration Assume $\kappa=0$. Initially we have to compute the $R Q$-factorization of the matrix $S$. One can compute this factorization similarly as the $Q R$-factorization, but now based on Givens transformations acting on the right.

First a Givens transformation acting on column 1 and 2 annihilating all elements in the first column below $s_{11}$ is applied. This transformation is followed by a Givens transformation acting on column 2 and 3, annihilating all elements in the second column below $s_{22}$. This procedure can easily be continued.

Thus applying $n-1$ Givens transformations on the right creates an upper triangular matrix $R$ in case of a $\{1\}$-semiseparable matrix. We obtain the following relation for a $\{1\}$-semiseparable matrix $S$ (see, e.g., $[27,28,25]) S=R Q$ with $Q$ a product of $n-1$ Givens transformations ${ }^{3}$.

\footnotetext{
3 We remark that other types of factorizations based on Gauss transforms can also be computed in a similar fashion, this results in $G R$-type of algorithms (see [27]).
} 
This leads to

$$
\begin{aligned}
S-\sigma I & =\left(S Q^{H}-\sigma Q^{H}\right) Q \\
& =\left(R-\sigma Q^{H}\right) Q .
\end{aligned}
$$

Due to the specific structure of the matrix $Q^{H}$, we can see that the matrix $\sigma Q^{H}$ will be a unitary Hessenberg matrix up to the scaling with $\sigma$.

Hence we obtain

$$
\begin{aligned}
S-\sigma I & =H Q \\
& =\hat{Q} \hat{R} Q .
\end{aligned}
$$

In the final step we need to compute the $Q R$-factorization of the intermediate matrix $H$. Similarly as described above, we can use $n-1$ Givens transformations, each transformation annihilating one subdiagonal element.

Hence, the unitary matrix used for determining the similarity transformation is the matrix $\hat{Q}$, which only consists of $n-1$ Givens transformations. Since the complexity of the iteration is determined by the factor $Q$ one might expect a lower complexity for performing one iteration step of the $Q R$-method driven by a rational function, w.r.t. the single shifted $Q R$-method. Remark, that we did not even discuss the enhanced convergence behavior due to the fact that rational iterates are performed. In [23] an implicit version of this method is presented and globally a speedup of $50 \%$ is achieved w.r.t. the single shifted $Q R$-method.

As mentioned before, the details on how to perform these operations effectively and in an implicit way will even not be described here, they would lead us to far from the general purpose of the manuscript. In a forthcoming manuscript (see [23]), we will discuss the issues such as:

- An effective computation of the $R Q$-factorization. Based on the Givens-weight representation of semiseparable matrices we can even omit this computation.

- An implicit implementation of the involved method.

- Detailed complexity counts and comparisons with the standard $Q R$-method for semiseparable matrices.

Even though not discussed in detail, these results can be generalized in a straightforward manner to the higher order case, i.e. the class of $\{p, q\}$-semiseparable matrices.

4.1.3 Preservation of the semiseparable structure We know that the semiseparable structure will be preserved under a $Q R$ and $R Q$-iterations [17] and hence also under the rational method presented here. Nevertheless, it is worth trying to prove the preservation of the structure directly by investigating in detail the structure of the intermediate matrices. A thorough investigation of these results is given in [23]. In this manuscript it is also shown that one can interpret the rational factorization differently. The unitary factor determining the similarity transformation can also be considered as coming from a $Q H$-factorization in which the matrix $H$ has the lower triangular part of semiseparable form. All these connections are explored in detail in [23].

\subsection{Semiseparable plus diagonal}

Semiseparable plus diagonal matrices appear in the analysis of orthogonal rational functions [29, 10]. Moreover, they can also be used as intermediate matrices when computing eigenvalues of symmetric matrices. Since the reduction of a symmetric matrix to a semiseparable one has advantageous convergence properties [30], the $Q R$-method for semiseparable plus diagonal matrices is also of interest.

We consider a semiseparable plus diagonal matrix $S+D$, with $D$ of diagonal form and $S$ of semiseparable form. Initially we need to compute the $R Q$ factorization:

$$
S+D-\kappa I=R Q=R Q_{1} Q_{2} .
$$


The unitary matrix $Q=Q_{1} Q_{2}$, consists of two sequences of Givens transformations, computed in an identical way as in the semiseparable case. The matrix $Q_{2}^{H}$ consists of $n-1$ Givens transformations, performed from left to right and annihilating almost an entire column in each operation. As a result we obtain $(S+D-\kappa I) Q_{2}^{H}=H$, with $H$ a Hessenberg matrix. The sequence of Givens transformations $Q_{1}^{H}$ transforms the matrix $H$ to upper triangular form. The matrix $Q_{1}^{H}$ also consists of $n-1$ Givens transformations, now performed from right to left.

Since computing an $R Q$-factorization is equally expensive as a $Q R$-factorization, the unitary factor in the $Q R$-factorization, which is used for performing a $Q R$-step consists of $2 n-2$ Givens transformations.

We will now show that the unitary factor for performing a step of a $Q R$-iteration driven by a rational function also consists of $2 n-2$ Givens transformations. Hence performing such a $Q R$ step might be equally expensive as performing a single shifted $Q R$-step in case of a semiseparable plus diagonal matrix.

The next step in computing the unitary factor determining the $Q R$-step driven by a rational function consists of computing the $Q R$-factorization of the matrix product $(S+D-\sigma I) Q^{H}$ :

$$
(S+D-\sigma I) Q^{H}=\left(R-(\sigma-\kappa) Q^{H}\right),
$$

since the matrix $Q$ consists of two sequences of Givens transformations, one can easily deduce that the matrix $\left(R-(\sigma-\kappa) Q^{H}\right)$, will have the lower triangular part also of semiseparable plus diagonal form. This means that the resulting matrix will have all subblocks taken out of the strictly lower triangular part of rank at most equal to 1 .

Hence computing the $Q R$-factorization of the matrix $\left(R-(\sigma-\kappa) Q^{H}\right)$ only involves $2 n-2$ Givens transformations. This means that the unitary similarity transformation determining the $Q R$ step driven by a rational function, has the same complexity as the unitary similarity transformation determining a single shifted $Q R$-step.

A similar remark as in the previous subsection holds. The results can be generalized to the higher order semiseparable plus diagonal case, as well as the higher order semiseparable plus band case. The results are similar, but involve more technical details.

\subsection{Unitary Hessenberg matrices}

The Schur parameter representation for unitary Hessenberg matrices is a widespread tool for dealing with these matrices (see $[31,32,33,34]$ ).

Suppose a unitary Hessenberg matrix $H \in \mathbb{C}^{n \times n}$ is given. An easy calculation reveals that this matrix can be written as the product of $n-1$ Givens transformations (this is the so-called Schur parameter representation):

$$
H=G_{1} G_{2} \ldots G_{n-1},
$$

where $G_{i}$ is a Givens transformation acting on columns (rows) $i$ and $i+1$.

Based on this representation one can see that the upper triangular part is of semiseparable form.

Based on this low rank part present in the upper triangular part of the unitary Hessenberg matrix and based on the results of semiseparable matrices presented before one can deduce a $Q R$-method driven by a rational function for lower unitary Hessenberg matrices. As a result one obtains a rational driven $Q R$-method ( $\sigma$ as shift, $\kappa=0$ ) involving a chasing procedure with $n-1$ Givens transformations, which is equally expensive as the standard bulge chasing method in the $Q R$-case [34].

Since the roots of the unitary Hessenberg matrix lie, however, on the unit circle the rational iteration with $\kappa=0$ does not create an enhanced convergence behavior. In fact both methods are in a certain sense dual to each other: equally expensive and equally fast. 


\subsection{Tridiagonal case}

Since the standard $Q R$-algorithm is mostly applied onto tridiagonal and Hessenberg matrices we will take a closer look at the structures involved when using tridiagonal matrices.

The $Q$-factor in the $Q R$-factorization of $T-\kappa I$ consists of a sequence of $n-1$ Givens transformations, each annihilating one subdiagonal element of the tridiagonal matrix $T-\kappa I$. Hence the unitary factor determining the similarity transformation consists of $n-1$ Givens transformations. We will show now that the unitary factor in the rational case is twice as complex, i.e., it consists of $2 n-2$ Givens transformations.

Initially one computes the $R Q$-factorization of the shifted tridiagonal matrix $T-\kappa I$ :

$$
(T-\kappa I)=R Q .
$$

Again, Givens transformations are used, $Q^{H}=G_{1}^{H} \ldots G_{n-1}^{H}$, where each Givens performed on the right annihilates a subdiagonal element from the symmetric tridiagonal matrix $T-\kappa I$. Givens transformation $G_{1}$ acts on column 1 and 2, Givens transformation $G_{2}$ acts on column 2 and 3, and so forth.

Continuing the new iteration process gives us the following equations:

$$
(T-\sigma I) Q=\left(R-(\sigma-\kappa) Q^{H}\right) Q .
$$

The unitary matrix determining the unitary similarity transformation is determined by the $Q R$ factorization of the matrix $\left(R-(\sigma-\kappa) Q^{H}\right)$. Since the matrix $Q^{H}$ consists of $n-1$ Givens transformations, we know from the previous subsection that this matrix is a lower unitary Hessenberg matrix. As a result the lower triangular part of $\left(R-(\sigma-\kappa) Q^{H}\right)$ satisfies the same structural constraints as the lower triangular part of a semiseparable plus diagonal matrix. Hence, computing the $Q R$-factorization of this matrix results in computing $2 n-2$ Givens transformations.

Hence the unitary matrix determining the new $Q R$-iteration consists of $2 n-2$ Givens transformations, which is twice as much as the number of Givens transformations in the unitary factor for the single shifted $Q R$-method. In a certain sense, this is not unlogical as the new iteration performs a $Q R$ and an $R Q$ step at once. Hence, for the tridiagonal the new iteration is twice as expensive as a single shifted $Q R$ or $R Q$-step.

\section{Conclusions \& Future work}

In this manuscript a new type of $Q R$-iteration was presented. The approach corresponds to a $Q R$ iteration driven by a rational function. The convergence properties as well as the relation with standard $Q R$-theory were discussed. Some examples were presented, showing that this approach does not necessarily lead to an increased complexity.

This manuscript opens several possibilities for further investigation. First there is the extension of this 'basic' rational step towards a more general 'easy computable' multishift setting allowing to compute efficiently the $Q R$-factorization of a general rational function in the matrix $A$.

There is also the search for other classes of matrices allowing an easy implementation of this rational $Q R$-algorithm. In a forthcoming manuscript an implicit version of this method is designed for computing eigenvalues of semiseparable matrices.

\section{References}

1. J. H. Wilkinson. The Algebraic Eigenvalue Problem. Numerical Mathematics and Scientific Computation. Oxford University Press, New York, USA, 1999.

2. B. N. Parlett. The Symmetric Eigenvalue Problem, volume 20 of Classics in Applied Mathematics. SIAM, Philadelphia, Pennsylvania, USA, 1998.

3. A. A. Dubrulle and G. H. Golub. A multishift $Q R$ iteration without computation of the shifts. Numerical Algorithms, 7(2-4):173-181, 1994.

4. G. H. Golub and C. F. Van Loan. Matrix Computations. Johns Hopkins University Press, Baltimore, Maryland, USA, third edition, 1996. 
5. D. S. Watkins and L. Elsner. Convergence of algorithms of decomposition type for the eigenvalue problem. Linear Algebra and its Applications, 143:19-47, 1991.

6. D. S. Watkins. QR-like algorithms - an overview of convergence theory and practice. In J. Renegar, M. Shub, and S. Smale, editors, The Mathematics of Numerical Analysis, volume 32 of Lectures in Applied Mathematics, pages 879-893. American Mathematical Society, Providence, Rhode Island, USA, 1996.

7. A. Ruhe. Rational Krylov sequence methods for eigenvalue computation. Linear Algebra and its Applications, 58:391-405, 1984.

8. A. Ruhe. Rational krylov algorithms for nonsymmetric eigenvalue problems, II: Matrix pairs. Linear Algebra and its Applications, 197/198:283-296, 1994.

9. A. Ruhe. The rational Krylov algorithm for nonsymmetric eigenvalue problems. III: Complex shifts for real matrices. BIT, 34:165-176, 1994.

10. D. Fasino. Rational Krylov matrices and QR-steps on hermitian diagonal-plus-semiseparable matrices. Numerical Linear Algebra with Applications, 12(8):743-754, October 2005.

11. D. S. Watkins. On the reduction of a Hamiltonian matrix to Hamiltonian Schur form. Electronic Transactions on Numerical Analysis, 23:141-157, 2006.

12. D. S. Watkins. Bidirectional chasing algorithms for the eigenvalue problem. SIAM Journal on Matrix Analysis and Applications, 14(1):166-179, 1993.

13. R. Byers. A hamiltonian $Q R$-algorithm. SIAM Journal on Scientific and Statistical Computation, 7(1):212-229, January 1986.

14. D. S. Watkins. Bulge exchanges in algorithms of QR-type. SIAM Journal on Matrix Analysis and Applications, 19(4):1074-1096, 1998.

15. J. H. Wilkinson. The algebraic eigenvalue problem. Oxford University Press, New York, USA, 1965.

16. F. R. Gantmacher and M. G. Kreı̌n. Oscillation Matrices and Kernels and Small Vibrations of Mechanical Systems. AMS Chelsea Publishing, Providence, Rhode Island, USA, revised edition, 2002.

17. R. Vandebril, M. Van Barel, and N. Mastronardi. An implicit $Q R$-algorithm for symmetric semiseparable matrices. Numerical Linear Algebra with Applications, 12(7):625-658, 2005.

18. Y. Eidelman, I. C. Gohberg, and V. Olshevsky. The QR iteration method for Hermitian quasiseparable matrices of an arbitrary order. Linear Algebra and its Applications, 404:305-324, July 2005.

19. D. A. Bini, F. Daddi, and L. Gemignani. On the shifted $Q R$ iteration applied to companion matrices. Electronic Transactions on Numerical Analysis, 18:137-152, 2004.

20. D. A. Bini, L. Gemignani, and V. Y. Pan. Fast and stable $Q R$ eigenvalue algorithms for generalized companion matrices and secular equations. Numerische Mathematik, 100(3):373-408, 2005.

21. D. A. Bini, Y. Eidelman, L. Gemignani, and I. C. Gohberg. Fast QR eigenvalue algorithms for Hessenberg matrices which are rank-one perturbations of unitary matrices. SIAM Journal on Matrix Analysis and Applications, 29(2):566-585, 2007.

22. D. S. Watkins. Understanding the $Q R$ algorithm. SIAM Review, 24(4):427-440, 1982.

23. R. Vandebril, M. Van Barel, and N. Mastronardi. A new iteration for computing the eigenvalues of semiseparable (plus diagonal) matrices. Technical Report TW507, Department of Computer Science, Katholieke Universiteit Leuven, Celestijnenlaan 200A, 3000 Leuven (Heverlee), Belgium, October 2007.

24. E. Van Camp, N. Mastronardi, and M. Van Barel. Two fast algorithms for solving diagonal-plus-semiseparable linear systems. Journal of Computational and Applied Mathematics, 164-165:731-747, 2004.

25. P. Dewilde and A.-J. van der Veen. Time-Varying Systems and Computations. Kluwer Academic Publishers, Boston, Massachusetts, USA, 1998.

26. Y. Eidelman and I. C. Gohberg. A modification of the Dewilde-van der Veen method for inversion of finite structured matrices. Linear Algebra and its Applications, 343-344:419-450, April 2002.

27. R. Vandebril, M. Van Barel, and N. Mastronardi. Matrix Computations and Semiseparable Matrices, Volume I: Linear Systems. Johns Hopkins University Press, Baltimore, Maryland, USA, 2008.

28. S. Delvaux and M. Van Barel. A QR-based solver for rank structured matrices. Technical Report TW454, Department of Computer Science, Katholieke Universiteit Leuven, Celestijnenlaan 200A, 3000 Leuven (Heverlee), Belgium, March 2006. To appear in SIMAX.

29. M. Van Barel, D. Fasino, L. Gemignani, and N. Mastronardi. Orthogonal rational functions and structured matrices. SIAM Journal on Matrix Analysis and Applications, 26(3):810-829, 2005.

30. R. Vandebril, E. Van Camp, M. Van Barel, and N. Mastronardi. On the convergence properties of the orthogonal similarity transformations to tridiagonal and semiseparable (plus diagonal) form. Numerische Mathematik, 104:205-239, 2006.

31. T. L. Wang, Z. J. and W. B. Gragg. Convergence of the shifted QR algorithm, for unitary Hessenberg matrices. Mathematics of Computation, 71(240):1473-1496, 2002.

32. T. L. Wang, Z. J. and W. B. Gragg. Convergence of the unitary Hessenberg QR algorithm with unimodular shifts. In English.

33. M. Stewart. Stability properties of several variants of the unitary Hessenberg QR-algorithm in structured matrices in mathematics. In V. Olshevsky, editor, Structured Matrices in Mathematics, Computer Science and Engineering, II, volume 281 of Contemporary Mathematics, pages 57-72. American Mathematical Society, Providence, Rhode Island, USA, 2001.

34. W. B. Gragg. The QR algorithm for unitary Hessenberg matrices. Journal of Computational and Applied Mathematics, 16:1-8, 1986. 